\title{
Primary Patency Rate of Superficial Femoral Artery Angioplasty in Patients with Stenosis/Occlusion of Femoropopliteal Artery
}

\author{
Pezhman Farshidmehr ${ }^{\circledR}$, Roozbeh Cheraghali $^{\circledR}$, Hossein Zabihi Mahmoud Abadi ${ }^{\circledR}$, Mohammad \\ Sadra Nazari ${ }^{1}$, Ali Gilani ${ }^{3}$, Ehsan Rahimpour ${ }^{3 *}$
}

${ }^{1}$ Department of Vascular Surgery, Sina Hospital, Tehran University of Medical Sciences, Tehran, Iran

${ }^{2}$ Golestan University of Medical Sciences, Gorgan, Iran

${ }^{3}$ Department of General Surgery, Sina Hospital, Tehran University of Medical Sciences, Tehran, Iran

*Corresponding Author: Ehsan Rahimpour, M.D., Assistant Professor of Surgery, Department of General Surgery, School of Medicine, Sina Hospital, Tehran University of Medical Sciences, Tehran, Iran. Email: dr.rahimpour83@gmail.com

Received September 21, 2020; Accepted December 27, 2020; Online Published January 8, 2021

\begin{abstract}
Background: The primary patency rate (PPR) is still controversial in angioplasty and heart bypass surgery for blood vessel repair and reconstruction in patients with femoropopliteal disease.

Objectives: This study aimed to investigate the prolonged PPR rate in patients with stenosis/occlusion of the femoropopliteal artery undergoing superficial femoral artery (SFA) and popliteal angioplasty.

Methods: A case series study population consisted of patients demonstrating femoropopliteal artery occlusion referred to Sina Hospital, Tehran, Iran for angiography during 2016-2018. After angiography, patients underwent either stent placement or balloon angioplasty in the case of stenosis/occlusion of femoropopliteal arteries. After angioplasty, patients were followed up in the 3rd, 12th, and 24th months for re-examination, and color Doppler ultrasonography of femoropopliteal arteries was also performed to measure the patency rate. The SPSS Statistics version 21.0 was used to analyze the data. The Kaplan-Meier method and a log-rank test were utilized to evaluate this rate.

Results: Sixty patients were included in the study, from which 44 were women $(73.3 \%)$ and 16 were men $(26.6 \%)$ with a mean age of 69.9 years. Fifty-two, 41, and 29 patients were examined at intervals of 3, 12, 24 months, with PPRs of $86 \%, 79 \%$, and $68 \%$, respectively. There was a significant relationship between claudication degree and procedure success $(P=0.02)$.

Conclusion: The prolonged PPR rate of patients after femoropopliteal artery angioplasty was acceptable and was a safe and effective treatment. For future studies, an increase in the number of study variables, a more comprehensive classification, and PPR-related variables are suggested.
\end{abstract}

Keywords: Angioplasty, Femoral Artery, Artery Occlusion, Primary Patency, Stenosis

\section{Background}

The primary patency rate (PPR) is still controversial in angioplasty and heart bypass surgery for blood vessel repair and reconstruction in patients with femoral-popliteal disease. ${ }^{1}$ Since half of the patients with peripheral artery disease have no clinical symptoms, detailed data are not available on the incidence and prevalence of the disease. Based on the related reports, the rate of stenosis/occlusion of femoropopliteal and iliac arteries is $41 \%$ and $23 \%$, respectively, which means the femoral artery is the most common site for stenosis/occlusion. Balloon angioplasty affects early occlusion only about $40 \%-60 \%$ after 6-12 months. ${ }^{2}$ The PPR rate depends on the disease stage, the severity of vascular occlusion, and runoff in below-theknee arteries. ${ }^{3}$

$\mathrm{CT}$ angiography and magnetic resonance angiography
(MRA) are two significant and sensitive imaging modalities to image blood vessels aimed at detecting the extent of vascular occlusion by atherosclerotic plaques. ${ }^{4}$ Contrast angiography is not usually applied for all patients unless treatment goals such as percutaneous transluminal angioplasty or stent placement are prescribed for the patient. Stent placement leads to solving vascular occlusion and, consequently, the open vascular lumen. PPR is the periodical evaluation of the degree of openness of the artery in which the stent is placed after stent placement. By calculating the rate of blood flow passing through the angioplasty arteries using the color doppler ultrasonography, we can examine the vascular PPR. ${ }^{5}$ Different statistics have been reported on prolonged PPR after repairing the femoropopliteal arteries; for example, three different values of $87.3 \%, 77 \%$, and $76 \%$ were

Copyright $($ C 2021 The Author(s). This is an open-access article distributed under the terms of the Creative Commons Attribution License (http:// creativecommons.org/licenses/by/4.0), which permits unrestricted use, distribution, and reproduction in any medium, provided the original work is properly cited. 
reported for one-year PPR..$^{6-8}$

\section{Objectives}

The present study aimed to investigate the prolonged PPR rate in patients with stenosis/occlusion of the femoropopliteal artery undergoing superficial femoral artery (SFA) and popliteal angioplasty.

\section{Methods}

The present research study is of case series type. The study population consisted of patients demonstrating femoropopliteal artery occlusion referred to the Division of Vascular and Endovascular surgery of Sina Specialized \& Subspecialty Hospital for angiography, Tehran, Iran during 2016-2018. The study sample size was calculated with an $81 \%$ average PPR rate (up to 12 months), a $10 \%$ difference, and a $5 \%$ coefficient of error.

The inclusion criteria were the age range of $40-80$ years and symptoms of vascular occlusion. The exclusion criteria were having rheumatic and background joint diseases (these groups of patients were excluded from the study due to possible muscle and joint pain that is confused with ischemic limb pain. After angiography, patients underwent angioplasty in terms of stenosis of femoropopliteal arteries. It should be noted that to prevent losses, people who could not be followed up in the future due to living in cities far from the hospital in question were also excluded from the study. Independent variables were smoking history, disease symptoms, associated morbidities, time for symptom onset until the time of first treatment, rate of arterial stenosis and occlusion, as well as anatomical location of stenosis, TASC II stratification. The dependent variables were the PPR rate and one-year prognosis in patients. After angiography, the patients with stenosis/occlusion of femoropopliteal arteries were included in the study, and then underwent the stent placement and/or balloon angioplasty. After angioplasty, patients were followed up in 3rd, 12th, and 24th months for re-examination, and color doppler ultrasonography of femoropopliteal arteries was also performed to measure the patency rate. Plavix was prescribed for pre- angioplasty patients. Antegrade or retrograde artery access was done under local anesthesia from the femoral artery on the same side (antegrade), or the femoral artery on the opposite side (retrograde) using a 4-Fr vascular sheath or a 6-Fr sheath, respectively. After artery access, 4000-8000 units of intravenous heparin were prescribed. Angioplasty was performed using a $4-5 \mathrm{~F}$ catheter passed through the stenosis/occlusion site using a wire to enter the runoff site. At this phase, the stent placement was performed in the case of failed initial angioplasty (the initial success criterion was persistent stenosis $>30$ ).

After angioplasty, patients received aspirin $80 \mathrm{mg} /$ day and Plavix $75 \mathrm{mg} /$ day along with statin for three months. It will take a long time to continue treatment with aspirin, cilostazol, and/or pentoxifylline. Patients were examined following angioplasty in the recovery room, a day later in the ward, and two weeks later. They were clinically examined at intervals of 3,12, 24 months after angioplasty to record the data including improvement in activity, walking, walk distance tolerance, changes compared to pre-procedure, distal pulse, wound healing, and control of lesions/wounds progression. The color doppler ultrasonography was also performed. The stenosis criterion was determined with peak systolic velocity (PSV) $>2.4$.

Patient information, including demographicinformation, examination results, laboratory test results, performed treatments, and other necessary information was filled in the information sheet designed for the present study. Patients with written informed consent were included in the study. No additional costs were imposed on patients, and no additional intervention was performed for patients.

The SPSS Statistics version 21.0 was used to analyze the data. The Kaplan-Meier method and a log-rank test were used to evaluate the PPR rate. The variables affecting the occurrence of complications and mortality following the stent placement were tested using a multivariate linear regression model.

\section{Results}

Sixty patients were included in the study, from which 44 were women $(73.3 \%)$ and 16 were men $(26.6 \%)$ with the mean age of 69.9 years. Fifty-two, 41, and 29 patients were examined at intervals of 3,12, and 24 months, with PPRs of $86 \%, 79 \%$, and $68 \%$, respectively. According to Table 1, 24 patients (45\%) had right-sided lesions, 22 (40\%) had left-sided lesions, and only 9 (15\%) had bilateral lesions. Eight patients died during the study period and 23 patients withdrew from the study.

Table 1. Descriptive Characteristics of Patients at the Beginning of Study

\begin{tabular}{|c|c|c|}
\hline $\begin{array}{l}\text { Risk Factors and Clinical Symptoms at the } \\
\text { Beginning }\end{array}$ & Number & Percent \\
\hline Smoking & 26 & 43.3 \\
\hline High blood pressure & 25 & 41.6 \\
\hline Diabetes & 41 & 69 \\
\hline Hyperlipidemia & 7 & 11.6 \\
\hline Heart failure & 6 & 10 \\
\hline Dialysis & 5 & 8.3 \\
\hline Claudication & 21 & 35 \\
\hline Pain during rest & 8 & 13.3 \\
\hline Gangrene & 13 & 21.6 \\
\hline Wounds & 20 & 33.3 \\
\hline \multicolumn{3}{|l|}{ Intensity of stenosis and obstruction } \\
\hline Stenosis and obstruction & 47 & 78.3 \\
\hline Stenosis & 8 & 13.3 \\
\hline Obstruction & 5 & 8.3 \\
\hline \multicolumn{3}{|l|}{ TASC } \\
\hline A & 27 & 45 \\
\hline B & 8 & 3.13 \\
\hline $\mathrm{C}$ & 9 & 15 \\
\hline $\mathrm{D}$ & 16 & 6.26 \\
\hline
\end{tabular}


Table 2 shows data relevant to the procedure success three months later concerning the risk factors. The chi-square test was used to investigate the relationship between postangioplasty factors and procedure success, which only a significant relationship was observed between claudication degree and procedure success $(P=0.02)$.

In the present study, the post-angioplasty PPR as the main variable, and factors affecting this parameter were evaluated.

Table 3 shows the frequency distribution of the patients according to the anatomical lesion classification system (TASC) 3 and 12 months after angioplasty. No significant relationship was observed between TASC and procedure success after three months $(P=0.47)$ and 12 months $(P=0.24)$.

\section{Discussion}

Findings indicated the post-angioplasty PPR rate of $86 \%$, $79 \%$, and $68 \%$ at intervals of 3,12 , and 24 months. After the first trimester, risk factors were not associated with the post-operative PPR rate. In a study by Clair et $\mathrm{al}^{9}$ the two-year PPR rate was $96.5 \%$. The $1 / 2 / 3 / 4$-year PPR rate was $81 \%, 74 \%, 68 \%$, and $65 \%$, respectively, in a study conducted by Iida et al. ${ }^{10}$ In addition, the $1 / 3 / 5$-year PPR rate was $81 \%, 61 \%$, and $58 \%$, respectively, in a study done by Capek et al. ${ }^{11}$

The PPR rate obtained in this study was acceptable compared to those reported in several studies. The value reported for the PPR rate in several studies was slightly

Table 2. Comparison of the Frequency Distribution of Success in Practice, 3 Months After Surgery According to Risk Factors

\begin{tabular}{lccc}
\hline & Open & Stenosis and occlude & P Value \\
\hline Smoking & $18(39.1 \%)$ & $5(83.33 \%)$ & 0.24 \\
High blood pressure & $19(41.3 \%)$ & $3(50 \%)$ & 0.98 \\
Diabetes & $29(63 \%)$ & $5(83.33 \%)$ & 0.81 \\
Hyperlipidemia & $5(10.8 \%)$ & $0(0 \%)$ & 0.87 \\
Heart failure & $4(8.6 \%)$ & $1(17 \%)$ & 0.94 \\
$\begin{array}{l}\text { Dialysis } \\
\text { Claudication after }\end{array}$ & $3(6.5 \%)$ & $2(33.33 \%)$ & 0.22 \\
angioplasty & $3(6.5 \%)$ & $3(50 \%)$ & 0.020 \\
\hline
\end{tabular}

Table 3. Comparison of the Frequency Distribution of Patients According to the Anatomical Lesion Classification System (TASC) 3 and 12 Months After Angioplasty

\begin{tabular}{rcccc}
\hline TASC & & Stenosis and Occlude & Open & P Value \\
\hline & A & $2(33.33 \%)$ & $23(50 \%)$ & \\
After 3 month & B & $2(33.33 \%)$ & $5(11 \%)$ & 0.47 \\
& C & $1(16.67 \%)$ & $6(13 \%)$ & \\
& D & $1(16.67 \%)$ & $12(26 \%)$ & \\
After 12 month & A & $3(37 / 5 \%)$ & $19(58 \%)$ & \\
& B & 0 & $5(15 \%)$ & \multirow{2}{*}{0.24} \\
& C & $2(25 \%)$ & $3(9 \%)$ & \\
\hline
\end{tabular}

higher. ${ }^{12}$ The PPR rate depends on different background and technical factors that may affect the success rate. The lesion length is important so that it is possible to fail arterial reconstruction in less time in the case of a longer lesion in the vein pathway. ${ }^{13}$ The matter of stent used also affects its durability so that nitinol stents are durable more than other types of metal. ${ }^{14-16}$ Socio-economic factors can also affect the severity of the disease as well as the post-treatment PPR rate, which were not examined in the present work. ${ }^{17,18}$ Procedure-related factors such as antiplaque treatment and distal collateral vessel status relative to lesion also affect the prolonged PPR rate. ${ }^{19,20}$ Each of the mentioned factors can be a reason for the difference between values reported for PPR in this study and other similar studies.

Among the factors affecting PPR, claudication was significant. In a study by Brewster et $\mathrm{al}^{21}$ five factors with a significant effect on prolonged PPR were identified through a 16-year investigation. The transplanted vein was the most crucial factor so that the vein transplant using a vein from the patient's own body had better-prolonged performance compared to other types of grafts. In the study performed by Capek et al, the crucial factors affecting the prognosis were diabetes and extensive atherosclerosis. In the present study, it was not possible to provide a significant relationship using the known variables and risk factors due to the small number of patients in the stenosis/occlusion group.

\section{Conclusion}

The prolonged PPR rate of patients after femoropopliteal artery angioplasty was acceptable and was a safe and effective treatment of femoropopliteal artery occlusion. It is recommended to investigate the PPR-related variables in depth by increasing the study sample size in future studies.

\section{Authors' Contributions}

All authors contributed equally to this study.

\section{Conflict of Interest Disclosures}

The authors declare that they have no conflicts of interest.

\section{Ethical Approval}

Current study was approved by Tehran University of Medical Sciences Ethics Committee.

\section{References}

1. Pereira CE, Albers $M$, Romiti $M$, Brochado-Neto FC, Pereira CA. Meta-analysis of femoropopliteal bypass grafts for lower extremity arterial insufficiency. J Vasc Surg. 2006;44(3):510517. doi:10.1016/j.jvs.2006.04.054.

2. Wilson NM, Chan O, Thomas ML, Browse NL. Intravenous digital subtraction angiography in the management of peripheral vascular disease. J Cardiovasc Surg (Torino). 1991;32(6):747-752.

3. Zwiebel WJ. Venous thrombosis. Introduction to Vascular Ultrasonography. 4th ed. Philadelphia: WB Saunders Company; 2000.

4. Sutton D. Text Book of Radiology and Imaging. New York: Churchill Livingstone; 2003. p. 428-430.

5. Ahmadi R, Ugurluoglu A, Schillinger $M$, Katzenschlager R, 
Sabeti S, Minar E. Duplex ultrasound-guided femoropopliteal angioplasty: initial and 12-month results from a case controlled study. J Endovasc Ther. 2002;9(6):873-881. doi:10.1177/152660280200900622.

6. Saxon RR, Coffman JM, Gooding JM, Ponec DJ. Longterm patency and clinical outcome of the Viabahn stentgraft for femoropopliteal artery obstructions. J Vasc Interv Radiol. 2007;18(11):1341-1349; quiz 1350. doi:10.1016/j. jvir.2007.07.011.

7. lida $\mathrm{O}$, Soga $\mathrm{Y}$, Hirano $\mathrm{K}$, et al. Long-term outcomes and risk stratification of patency following nitinol stenting in the femoropopliteal segment: retrospective multicenter analysis. J Endovasc Ther. 2011;18(6):753-761. doi:10.1583/11-3581.1.

8. Aihara $\mathrm{H}$, Soga $\mathrm{Y}$, lida $\mathrm{O}$, et al. Long-term outcomes of endovascular therapy for aortoiliac bifurcation lesions in the real-Al registry. J Endovasc Ther. 2014;21(1):25-33. doi:10.1583/13-4410mr.1.

9. Clair DG, Adams J, Reen B, et al. The EPIC nitinol stent system in the treatment of iliac artery lesions: one-year results from the ORION clinical trial. J Endovasc Ther. 2014;21(2):213-222. doi:10.1583/13-4560.1.

10. Iida O, Nanto S, Uematsu M, Ikeoka K, Okamoto S, Nagata $S$. Influence of stent fracture on the long-term patency in the femoro-popliteal artery: experience of 4 years. JACC Cardiovasc Interv. 2009;2(7):665-671. doi:10.1016/j.jcin.2009.04.014.

11. Capek P, McLean GK, Berkowitz HD. Femoropopliteal angioplasty. Factors influencing long-term success. Circulation. 1991;83(2 Suppl):I70-80.

12. Söder HK, Manninen HI, Räsänen HT, Kaukanen E, Jaakkola P, Matsi PJ. Failure of prolonged dilation to improve longterm patency of femoropopliteal artery angioplasty: results of a prospective trial. J Vasc Interv Radiol. 2002;13(4):361-369. doi:10.1016/s1051-0443(07)61737-0.

13. Kim SJ, Kim W, Kim JB, et al. Determinants of procedural success and patency following subintimal angioplasty in patients with TASC C and D femoropopliteal arterial disease.
Circ J. 2010;74(9):1959-1964. doi:10.1253/circj.cj-10-0106.

14. Scheinert D, Scheinert S, Sax J, et al. Prevalence and clinica impact of stent fractures after femoropopliteal stenting. J Am Coll Cardiol. 2005;45(2):312-315. doi:10.1016/j. jacc.2004.11.026.

15. Soga $Y$, lida O, Hirano K, Yokoi H, Nanto S, Nobuyoshi M Mid-term clinical outcome and predictors of vessel patency after femoropopliteal stenting with self-expandable nitino stent. J Vasc Surg. 2010;52(3):608-615. doi:10.1016/j. jvs.2010.03.050

16. Sabeti S, Mlekusch W, Amighi J, Minar E, Schillinger M Primary patency of long-segment self-expanding nitinol stents in the femoropopliteal arteries. J Endovasc Ther. 2005;12(1):612. doi:10.1583/04-1359.1.

17. Kröger K, Dragano N, Stang A, et al. An unequal social distribution of peripheral arterial disease and the possible explanations: results from a population-based study. Vasc Med. 2009;14(4):289-296. doi:10.1177/1358863x09102294.

18. Durham CA, Mohr MC, Parker FM, Bogey WM, Powell CS, Stoner MC. The impact of socioeconomic factors on outcome and hospital costs associated with femoropopliteal revascularization. J Vasc Surg. 2010;52(3):606-607. doi:10.1016/j.jvs.2010.04.011.

19. Ullery BW, Tran K, Itoga N, Casey K, Dalman RL, Lee JT. Safety and efficacy of antiplatelet/anticoagulation regimens after Viabahn stent graft treatment for femoropopliteal occlusive disease. J Vasc Surg. 2015;61(6):1479-1488. doi:10.1016/j. jvs.2014.12.062.

20. Johnston PC, Vartanian SM, Runge SJ, et al. Risk factors for clinical failure after stent graft treatment for femoropopliteal occlusive disease. J Vasc Surg. 2012;56(4):998-1007. doi:10.1016/j.jvs.2012.03.010.

21. Brewster DC, LaSalle AJ, Robison JG, Strayhorn EC, Darling RC. Factors affecting patency of femoropopliteal bypass grafts. Surg Gynecol Obstet. 1983;157(5):437-442. 